Research Article

\title{
Different Forms of Ursolic Acid and Their Effect on Liver Regeneration
}

\author{
Lenka Žaloudková $\mathbb{D}^{\mathrm{D}},{ }^{1}$ Alena Tichá, ${ }^{1}$ Jana Nekvindová, ${ }^{1}$ Ladislava Pavlíková, ${ }^{1}$ \\ Zdeněk Zadák, ${ }^{2}$ and Pavel Živný ${ }^{3}$ \\ ${ }^{1}$ Institute of Clinical Biochemistry and Diagnostics, University Hospital Hradec Kralove, Sokolska Str. 581, \\ Hradec Kralove 500 05, Czech Republic \\ ${ }^{2}$ Department of Research and Development, University Hospital Hradec Kralove, Sokolska Str. 581, Hradec Kralove 500 05, \\ Czech Republic \\ ${ }^{3}$ 2nd Department of Internal Medicine-Gastroenterology, Charles University, Faculty of Medicine in Hradec Kralove, \\ Hradec Kralove 500 03, Czech Republic
}

Correspondence should be addressed to Lenka Žaloudková; lenka.zaloudkova@fnhk.cz

Received 20 February 2020; Revised 25 May 2020; Accepted 7 July 2020; Published 26 July 2020

Academic Editor: Susana Gorzalczany

Copyright (C) 2020 Lenka Žaloudková et al. This is an open access article distributed under the Creative Commons Attribution License, which permits unrestricted use, distribution, and reproduction in any medium, provided the original work is properly cited.

\begin{abstract}
The aim of this study was to determine the effect of natural and encapsulated sources of ursolic acid on liver regeneration. Four ursolate sources were tested. Two forms of ursolic acid encapsulates were combined with cyclodextrins, i.e., gamma-CD (gCD) and beta-CD, and two natural sources were adjusted by homogenization (HAP) and micronization of apple peel using Jonagold apples. All ursolate forms were applied intragastrically in daily doses of $20 \mathrm{mg}$ for 7 days. Laboratory rats were fed with standard laboratory diet. Further, gCD and MAP were also tested with a high-fat diet (6 weeks). Partial hepatectomy (PH) was performed 24 hours before the end of the experiment. The concentration of plasma hepatocyte growth factor (HGF) was determined with an immunoassay; simultaneously, the expression of HGF and CYP7A1 in the liver was quantified through qPCR. HGF expression and plasma levels were significantly increased 24 hours after $\mathrm{PH}$ in both the HAP $(p=0.038)$ and $\operatorname{HFgCD}$ groups $(p=0.036)$, respectively. The correlation between HGF expression and plasma values was significant $(p=0.04)$. The positive effects on liver regeneration were found in both the gCD and HAP forms of ursolic acid, whose effects were confirmed through the upregulation of HGF.
\end{abstract}

\section{Introduction}

Ursolic acid is classified within the group of triterpenes which, along with steroids, belongs to the group of isoprenoids whose biosynthesis is based on squalene. To date, $>1,000$ triterpenoids with an incredible range of biological activities have been isolated from natural sources. Among these, ursolic acid has biological properties such as an antiinflammatory, anticancer, antiangiogenic, and antioxidative agent $[1,2]$.

Ursolic acid (UA) is a pentacyclic triterpenoid found in several herbs, spices, and fruits like rosemary, thyme, apple, and olive. [3]. The consumption of apples has been linked to the prevention of various chronic disease, and apple peel exhibits more potent antioxidant and antiproliferative activities than apple flesh. Apple peel is a suitable source of UA, and these fruits are well available locally. Triterpenes content in various fruits and plants was confirmed the highest UA contain with lavender, rosemary, and apples, especially in the Jonagold variety [3]. The bioavailability of ursolic acid is problematic due to its hydrophobic characteristics. Its encapsulation with cyclodextrins or its increased bioavaibility through micronization has facilitated the intake of ursolic acid. In this regard, most researchers use organic solvents (e.g., acetone, ethanol, and dichloromethane) to dissolve ursolic acid or used organic solvents during preparation, 
which may be hazardous to humans. Further, its bioavailability has been assessed in the form of ursolic acid stearoyl glucoside [4], different phospholipid complexes [5], or nanoparticles [6]. When UA is combined with cyclodextrins, its solubility in water is greatly enhanced by the cyclic structure of the latter [7]. Inspite of possible bioavailability improvement, we have attempted to increase water solubility of ursolic acid by encapsulation. Cyclodextrins are often used in the pharmacology and food industry as additive compounds.

Partial hepatectomy $(\mathrm{PH})$ has been shown as the best method to test liver regeneration [8-10]. After the procedure, the remaining liver tissue is exposed to increased blood flow, and cell replication begins after 24 hours. Immediately after resection, the expression of urokinase plasminogen activator (uPA) is upregulated throughout the entire liver tissue, starting as early 5 minutes after $\mathrm{PH}$, activating the expression of matrix remodelling proteins such as the hepatocyte growth factor (HGF) [11]. The latter is a direct mitogen capable of inducing liver proliferation in an independent manner; however, there are other proteins classified as auxiliary liver mitogens (e.g., TNF alpha, IL-6) $[8,9]$. HGF is crucial for hepatic regeneration $[8,10]$; on the other hand, the effect of IL6 in hepatic regeneration has not been confirmed so far [12].

The aim of this study was to verify the effect that both natural and encapsulated sources of ursolic acid have on liver regeneration as well as to confirm a reliable biochemical blood marker indicating liver regeneration.

\section{Study Design}

2.1. Animals. Male Wistar rats (220-320 g in weight; 7 weeks of age) were purchased from BioTest (Konarovice, Czech Republic). The animals were housed at $22 \pm 2^{\circ} \mathrm{C}, 55 \pm 10 \%$ humidity, and 12 hours light/dark cycles. The animals had access to water and standard laboratory pellet diet ad libitum (Velaz, Czech Republic). The study protocols were approved by the Ethical Expert Committee of the Medical Faculty, Charles University Hradec Kralove (protocol No. MSMT42093/2015-5). All experiments were in adherence to rule No. 246/1992 for the protection of animals against cruelty. The rats were divided into 7 groups ( $n=6$ per group) after 1 week of acclimatization.

\subsection{Animal Groups}

CTRL: standard pellet diet for 7 days-Control group bCD: standard pellet diet for 7 days and beta-cyclodextrine ursolic acid

gCD: standard pellet diet for 7 days and gammacyclodextrine ursolic acid

HAP: standard pellet diet for 7 days and homogenate apple peel

MAP: standard pellet diet for 7 days and micronized apple

HFgCD: HF diet for 7 days and gamma-cyclodextrine ursolic acid

HF-MAP: HF diet for 7 days and micronized apple peel
2.3. Tested Compounds. Four ursolate substances were prepared from apple peel using Jonagold variety apples. Two substances were encapsulated with cyclodextrins (CD), gamma-CD (gCD), and beta-CD (bCD). The cyclodextrin (CD) encapsulates of ursolic acid were supplied by Betulinies (Stribrna Skalice, Czech Republic). The other two substances were adjusted by homogenization (HAP; $\varnothing>5 \mu \mathrm{m})$ and micronization of apple peel (MAP; $\varnothing=5 \mu \mathrm{m}$ ) [5] and were prepared by K2pharm (Opava, Czech Republic) without using organic solvents. All forms were supplemented on a daily basis as water suspensions directly into the stomach using a gastric tube (20 mg of UA per animal). The amount of ursolic acid was determined and verified after extraction of apple peel into ethylacetate by using GC-MS [13], and the amount of ursolic acid was $5.6 \mathrm{~g}$ in $100 \mathrm{~g}$ of Jonagold variety apple peel.

2.4. Diet. The animals were fed with a standard diet for 1 week before the study and then divided into 7 groups at random ( $n=6$ per group). The standard diet contained $21.1 \%$ protein, $5.1 \%$ fat, $60.0 \%$ carbohydrates, $3.9 \%$ fiber, $7.9 \%$ minerals, and $2.0 \%$ vitamins.

A high-fat diet (HFD) was prepared with $35.5 \%$ fat (beef tallow), 59\% protein and carbohydrates, and 5.5\% minerals and vitamins (Weber, Czech Republic). The corresponding animal groups were fed with this high-fat diet for 6 weeks before experiment.

2.5. Experimental Design. Partial hepatectomy (PH) (70\%) was practiced 24 hours before the end of the experiment according to Higgins and Anderson [14]. The animals were anesthetized using ether inhalation before surgery and placed in cages upon awakening. The last doses of ursolic acid were supplemented after PH. The animals were later overdosed with an ether anaesthetic 24 hours after $\mathrm{PH}$ and sacrificed by exsanguination from the abdominal aorta. The liver lobes were excised during partial hepatectomy and, the liver remnants were weighed, frozen, and stored for posterior analysis.

\section{Methods}

Blood samples were collected into a heparin-coated tube from the retro-orbital sinus before the administration of ursolic acid and before PH (Sarstedt, Germany). Blood samples were also collected at the end of the experiment. The blood cells were precipitated by centrifugation, and aliquots of plasma were stored at $-20^{\circ} \mathrm{C}$ for posterior analysis. The concentration of aspartate aminotransferase (AST), alanine aminotransferase (ALT), total cholesterol $(\mathrm{tCH})$, and triglyceride (TAG) was measured using a Cobas 8000 instrument (Roche, Basel). The concentration of hepatocyte growth factor (HGF) in plasma was determined with the sandwich enzyme immunoassay cloud kit (Clone Corporation, USA). Total bile acid (BA) was determined with an enzymatic cycling method (Diazyme Laboratories, USA). Liver weight was calculated by the following equation [15]: 


$$
\text { hepatic regeneration rate }(\mathrm{HRR})(\%)=\frac{C-(A-B)}{A \times 100},
$$

where $A$ is the estimated total liver weight at the time of the partial hepatectomy, $B$ is the excised liver weight, and $C$ is the weight of the regenerated liver at sacrifice.

3.1. RNA Isolation and $q P C R$. Total RNA was extracted from $\sim 30 \mathrm{mg}$ of snap frozen liver tissue samples using TRIzol (Invitrogen, USA). RNA concentration was determined with a Nanodrop ND1000 spectrophotometer (Thermo Scientific, USA) at $260 / 280 \mathrm{~nm}$. RNA integrity was determined with an Agilent bioanalyzer 2100 and RNA 6000 Nano kit (Agilent Technologies, USA). cDNA was synthesized using the Transcriptor First-Strand cDNA Synthesis kit (Roche) using $1 \mu \mathrm{g}$ of total RNA and a $1: 2$ ratio of dT and random hexamer primers according to the manufacturer's protocol. Subsequently, $25 \mathrm{ng}$ cDNA was included in the qPCR reaction using the 1X Gene Expression Master Mix and 1X TaqManlabeled primers (Table 1); the PCR reaction was performed in a RotorGene $Q$ real-time PCR instrument (Qiagen, Netherlands). The housekeeping gene HPRT1 was used as an expression reference. The standardized data were used to calculate relative gene expressions using the $2^{-\Delta \Delta \mathrm{CT}}$ method [16].

3.2. Statistical Analysis. Analyses were performed with the SigmaStat Software (Systat, USA). First, normality was tested by the Kolmogorov-Smirnov test. In normal data, comparisons were made among the groups using one-way ANOVA followed by Tukey-Kramer's post hoc test. The results are expressed as the mean $\pm \mathrm{SD}$. In the case of nonGaussian distribution, nonparametric Kruskal-Wallis test and Dunn's post hoc test were used. Data are expressed as median values (interquartile range). Significance was set at $p<0.05$.

\section{Results}

The plasma markers of lipid metabolism and liver enzyme activity were evaluated before administration of UA and after the administration of UA and after $\mathrm{PH}$ (Table 2). The concentrations of plasma TAG and tCH were significantly reduced following the administration of ursolate compounds in the HF diet group (Table 2). Further, the catalytic activity of ALT and AST confirmed that all UA forms result in the same level of activity in comparison with the CTRL and did not induce liver damage.

The level of $\mathrm{tCH}$ after $\mathrm{PH}$ (Table 2) was significantly higher in the HF diet groups. The remaining groups did not show hepatic impairment and the lipid metabolism was not different either except for the HF-MAP group where ALT activity was significantly increased. Moreover, significantly increased concentrations of $\mathrm{tCH}$ and TAG were observed in the plasma of the HF diet groups after $\mathrm{PH}$ in comparison with the CTRL. HGF expression in the liver and plasma levels is shown in Table 3. CYP7A1 expression in the liver
TABle 1: Primers.

\begin{tabular}{lcc}
\hline Assay ID & Gene & Gene name \\
\hline Rn00566673_m1 & Hgf & $\begin{array}{c}\text { Hepatocyte growth factor } \\
\text { Cytochrome P450, family 7, } \\
\text { subfamily a, polypeptide 1 }\end{array}$ \\
\hline
\end{tabular}

and plasma levels of BA are shown in Table 4. The Spearman correlation is shown in Table 5 and Figure 1.

HGF expression and plasma levels were significantly increased 24 hours after $\mathrm{PH}$ in the HAP and HFgCD groups (Table 3). The correlation between HGF expression in liver and plasma levels was confirmed by Spearman's correlation $(p<0.05)$ (Table 5, Figure 1). HGF expression and plasma levels are in agreement with HRR. Further, the levels of BA were lower in the MAP and HF-MAP groups (Table 4); however, it did not block the BA flow.

\section{Discussion}

In the present study, we evaluated the effect of UA on liver regeneration after its administration in various forms and without the use of an organic solvent adjuvant. The effects of UA cyclodextrins and UA micronization were evaluated under normal and high-fat diets, finding significantly increased levels of in TAG and $\mathrm{tCH}$ in the plasma of the evaluated rats in the latter condition (Table 2). Our data clearly showed (Table 2) that, after 6 weeks, HFD TAG and $\mathrm{tCH}$ were increased in plasma; however, there was a significant decrement in these levels after 7 days of UA administration. These results are consistent with the known hypolipidemic effects of UA $[17,18]$ in mice. In addition, no liver damage was caused by the tested UA sources as shown by the enzyme activity levels of ALT and AST after the administration of UA and after partial hepatectomy.

Significantly lower levels of TAG in rats after the administration of ursolic acid could be caused by an increased lipoprotein lipase activity; despite an HF diet, these decreased TAG and tCH levels could be observed during the 7 days of gCD and MAP administration (Table 2), which was confirmed in all of the experimental groups. Further, liver $\mathrm{tCH}$ was increased after $\mathrm{PH}$ due to the changes in energy available for cell proliferation. The HFgCD and HF-MAP groups showed a significantly higher concentration of $\mathrm{tCH}$ and TAG in plasma such as HGF expression, supporting the hypothesis that early lipid accumulation in the liver after $\mathrm{PH}$ could be beneficial for its regeneration $[19,20]$.

DNA replication was markedly reduced after $\mathrm{PH}$ while BA flow was blocked. Plasma HGF values were also decreased in the absence of $\mathrm{BA}$; on the other hand, HGF concentration was increased when BA flow was restored $[8,9,12]$. The levels of bile acids (BA) in our study groups were similar with significant differences only a diet with MAP or HF-MAP (Table 4). This indicates minimal BA liver load after $\mathrm{PH}$, showing no blockage of BA outflow in either study group.

The ursolic acid preparations confirmed increased liver regeneration in comparison to the CTRL group. HGF 


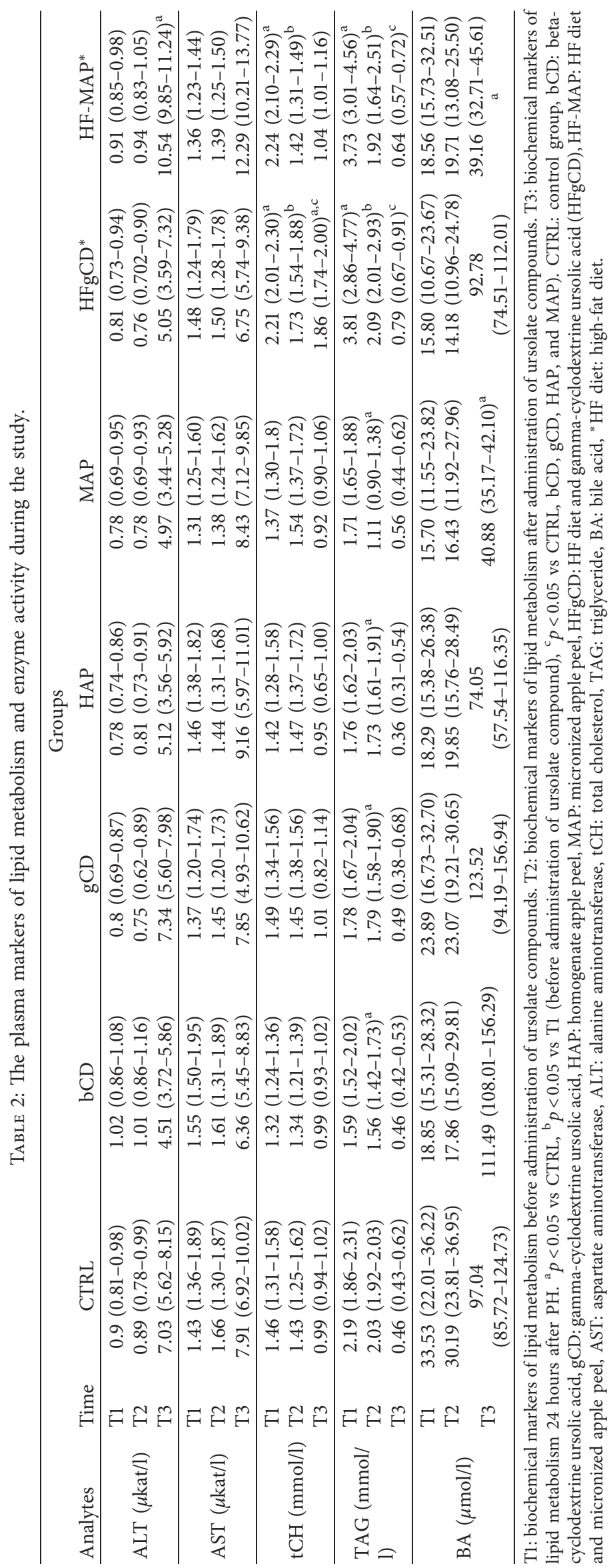


TABLE 3: HGF expression in the liver and concentration in plasma after PH and HRR.

\begin{tabular}{lccccccc}
\hline Group & CTRL & bCD & gCD & HAP & MAP & HFgCD & HF-MAP \\
\hline \multirow{2}{*}{ HGF (ng/l) in blood } & 31.46 & 43.58 & 55.76 & 62.67 & 43.56 & 63.58 & 41.96 \\
& $(25.05-42.82)$ & $(36.22-48.06)$ & $(41.91-59.83)$ & $(57.11-68.52)^{\mathrm{a}}$ & $(34.36-49.62)$ & $(56.88-78.83)^{\mathrm{a}}$ & $(34.04-52.87)$ \\
HGF expression [16] & 1.108 & 1.097 & 1.260 & 1.975 & 1.037 & 1.341 & 2.178 \\
HRR (\%) [15] & $(1.09-1.203)$ & $(1.02-1.193)$ & $(1.16-1.315)$ & $(1.815-2.109)^{\mathrm{a}}$ & $(0.987-1.126)$ & $(1.256-1.497)^{\mathrm{a}}$ & $(1.893-2.226)^{\mathrm{a}}$ \\
\hline
\end{tabular}

${ }^{a} p<0.05$ vs CTRL, HGF: hepatocyte growth factor, hepatic regeneration rate (HRR) (\%): $[C-(A-B)] / A \times 100 . A$ : estimated total liver weight at the time of the partial hepatectomy, $B$ : the excised liver weight, and $C$ : the weight of the regenerated liver at sacrifice.

TABLE 4: CYP7A1 expression in the liver and bile acid concentration in plasma after PH.

\begin{tabular}{lccccccc}
\hline Group & CTRL & bCD & gCD & HAP & MAP & HFgCD & HF-MAP \\
\hline BA after PH & 97.04 & 111.49 & 123.52 & 74.05 & 40.88 & 92.78 & 39.16 \\
$(\mu$ mol/l) & $(85.72-124.73)$ & $(108.01-156.29)$ & $(94.19-156.94)$ & $(57.54-116.35)$ & $(35.17-42.10)^{\mathrm{a}}$ & $(74.51-112.01)$ & $(32.71-45.61)^{\mathrm{a}}$ \\
CYP7A1 & 0.535 & 0.751 & 0.543 & 0.852 & 0.769 & 0.885 & 0.370 \\
expression [16] & $(0.397-0.672)$ & $(0.566-0.838)$ & $(0.409-0.596)$ & $(0.767-0.912)$ & $(0.627-0.803)$ & $(0.639-0.916)$ & $(0.315-0.458)$ \\
\hline
\end{tabular}

${ }^{a} p<0.05$ vs CTRL, CYP7A1: cytochrome P450, family 7 , subfamily a, polypeptide 1 .

TABLE 5: Spearman correlation of HGF expression in the liver and plasma levels.

\begin{tabular}{lcc}
\hline Group & $\mathrm{R}$ & $p<0.05$ \\
\hline HGF ELISA vs. HGF expression & 0.379 & 0.04 \\
CYP7A1 vs. BA & -0.0484 & 0.78 \\
\hline
\end{tabular}

HGF: hepatocyte growth factor, CYP7A1: cytochrome P450, family 7 , subfamily a, polypeptide $1, R$ : correlation coefficient.

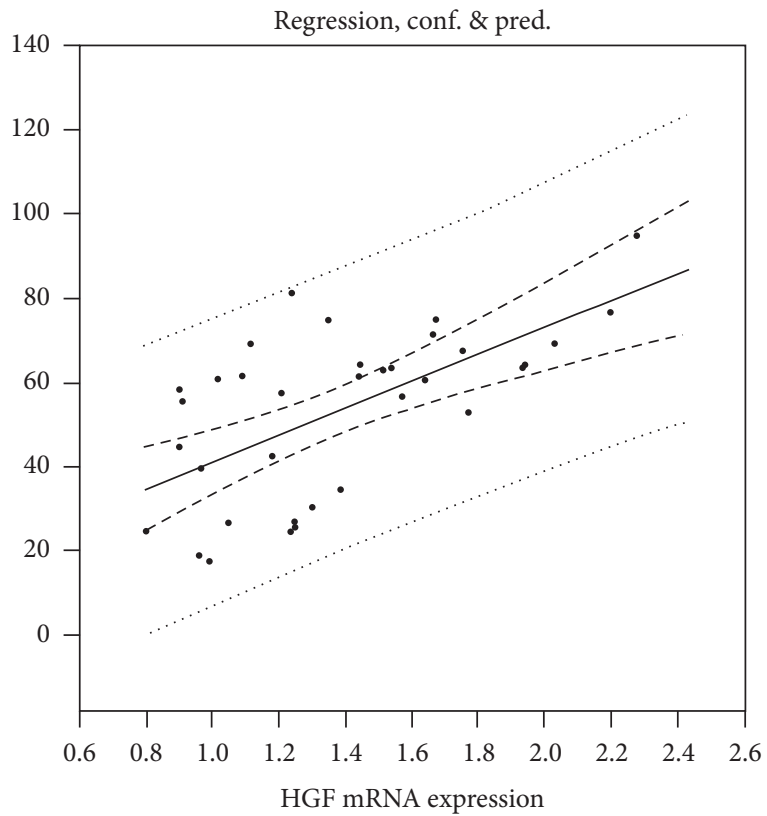

Figure 1: Spearman correlation of HGF expression in the liver and plasma levels.

expression in liver tissue and plasma levels were significantly increased 24 hours after $\mathrm{PH}$ in the HAP and HFgCD groups compared with those of the CTRL group, with both values being significant. HGF expression in the liver confirmed a significant increment in HF-MAP. The results (Table 3) are consistent with the calculated liver regeneration [15], and increased HRR in the HF diet may also be related to fat accumulation [20] in the liver. When comparing groups with the SLD diet, the results of both plasma HGF and hepatic expression HGF and HHR are consistent with those of [21], which administered ursolic acid as a chemical substance, observing a significant liver to body weight increment along with the upregulation of cyclins and C/EBP proteins [21].

The correlation results of plasma HGF and HGF expression are shown in Table 5 and Figure 1. We suggest that plasma HGF is a suitable marker indicating the rate of liver regeneration, which can be easily monitored through an ELISA. The biological activity of the tested forms of UA showed HAP as the most suitable source according to HGF expression, although gCD also showed acceptable results. It can be assumed that the homogenized peel of Jonagold apples is the most suitable source of ursolic acid without the need of toxic chemical solvents. Taken together, our findings are consistent $[8,9,21]$ and show $\mathrm{HGF}$ as a mitogenic substance with major effects on liver growth. We have shown that ursolic acid stimulates DNA synthesis, and at the same time, it displays hepatoprotection against apoptosis induce by high HGF levels.

\section{Conclusion}

The effects of ursolic acid extracted, micronized, and homogenized from apple peel on liver regeneration has not been previously described elsewhere. Interestingly, this ursolic acid extract had the same effect on liver regeneration observed with encapsulated gamma cyclodextrins. Therefore, suitable sources of ursolic acid for liver regeneration can now be extracted without the need of organic solvents. In addition, this study revealed that plasma HGF is a suitable biochemical marker of liver regeneration.

\section{Abbreviations}

ALT: Alanine aminotransferase

AST: Aspartate aminotransferase

BA: $\quad$ Bile acid

bCD: Beta-cyclodextrin ursolic acid 
CD: $\quad$ Cyclodextrines

C/EBP: CCAAT/enhancer binding proteins

CTRL: Control group

CY7A1: Cytochrome P450, family 7, subfamily a, polypeptide 1

gCD: Gamma-cyclodextrin ursolic acid

HAP: Homogenate apple peel

HF: High-fat diet

HFgCD: HF diet and gamma-cyclodextrin ursolic acid

HF- HF diet and micronized apple peel

MAP:

HGF: Hepatocyte growth factor,

HRR: Hepatic regeneration rate

MAP: $\quad$ Micronized apple

PCR: Polymerase chain reaction

PH: Partial hepatectomy

R: $\quad$ Correlation coefficient

TAG: Triglyceride

tCH: Total cholesterol

UA: Ursolic acid.

\section{Data Availability}

The data used to support the findings of this study are presented within the article.

\section{Disclosure}

The funding organization had no role in the experimental design, data collection and analysis, decision to publish, or the preparation of the paper.

\section{Conflicts of Interest}

The authors declare that they have no conflicts of interest.

\section{Acknowledgments}

This study was supported by the grant $\mathrm{MH} \mathrm{CZ-DRO}$ (UHHK, 00179906). The authors wish to thank Daniel Diaz, Ph.D., for proofreading this manuscript.

\section{References}

[1] L. Zhang, X. Huang, Z. Meng et al., "Significance and mechanism of CYP7al gene regulation during the acute phase of liver regeneration," Molecular Endocrinology, vol. 23, no. 2, pp. 137-145, 2009.

[2] J. P. Wu, C. C. Tsai, Y. L. Yeh et al., "Silymarin accelerates liver regeneration after partial hepatectomy," Evidence-Based Complementary and Alternative Medicine, vol. 2015, pp. 1-14, 2015.

[3] S. Jäger, H. Trojan, T. Kopp, M. Laszczyk, and A. Scheffler, "Pentacyclic triterpene distribution in various plants-rich sources for a new group of multi-potent plant extracts," Molecules, vol. 14, no. 6, pp. 2016-2031, 2009.

[4] I. Kazmi, M. Afzal, S. Rahman, M. Iqbal, F. Imam, and F. Anwar, "Antiobesity potential of ursolic acid stearoyl glucoside by inhibiting pancreatic lipase," European Journal of Pharmacology, vol. 709, no. 1-3, pp. 28-36, 2013.
[5] S. Biswas, P. K. Mukherjee, R. K. Harwansh, S. Bannerjee, and P. Bhattacharjee, "Enhanced bioavailability and hepatoprotectivity of optimized ursolic acid-phospholipid complex," Drug Development and Industrial Pharmacy, vol. 45, no. 6, pp. 946-958, 2019.

[6] L. Yang, Z. Sun, Y. Zu et al., "Physicochemical properties and oral bioavailability of ursolic acid nanoparticles using supercritical anti-solvent (SAS) process," Food Chemistry, vol. 132, no. 1, pp. 319-325, 2012.

[7] S. Carneiro, F. Costa Duarte, L. Heimfarth et al., "Cyclodextrin-drug inclusion complexes: In vivo and in vitro approaches," International Journal of Molecular Sciences, vol. 20, no. 3, p. 642, 2019.

[8] G. K. Michalopoulos, "Liver regeneration after partial hepatectomy," The American Journal of Pathology, vol. 176, no. 1, pp. 2-13, 2010.

[9] D. A. Rudnick and N. O. Davidson, "Functional relationships between lipid metabolism and liver regeneration," International Journal of Hepatology, vol. 2012, pp. 1-8, 2012.

[10] N. Fausto, A. D. Laird, and E. M. Webber, "Liver regeneration. 2. Role of growth factors and cytokines in hepatic regeneration," The FASEB Journal, vol. 9, no. 15, pp. 1527-1536, 1995.

[11] B. Delgado-Coello, M. A. Briones-Orta, M. Macías-Silva, and J. Mas-Oliva, "Cholesterol: recapitulation of its active role during liver regeneration," Liver International, vol. 31, no. 9, pp. 1271-1284, 2011.

[12] W. E. Naugler, "Bile acid flux is necessary for normal liver regeneration," PLoS One, vol. 9, no. 5, Article ID e97426, 2014.

[13] P. Šiman, A. Filipová, A. Tichá, M. Niang, A. Bezrouk, and R. Havelek, "Effective method of purification of betulin from birch bark: The importance of its purity for scientific and medicinal use," PLoS One, vol. 11, no. 5, Article ID e0154933, 2016.

[14] G. M. Higgins and R. M. Anderson, "Experimental pathology of the liver - restoration of the liver of the white rat following partial surgical removal," Archives of Pathology, vol. 12, pp. 186-202, 1931.

[15] M. Selzner and P.-A. Clavien, "Failure of regeneration of the steatotic rat liver: Disruption at two different levels in the regeneration pathway," Hepatology, vol. 31, no. 1, pp. 35-42, 2000.

[16] K. J. Livak and T. D. Schmittgen, "Analysis of relative gene expression data using real-time quantitative PCR and the $2^{-\Delta \Delta \mathrm{CT}}$ method," Methods, vol. 25, no. 4, pp. 402-408, 2001.

[17] B. Jayaprakasam, L. K. Olson, R. E. Schutzki, M.-H. Tai, and M. G. Nair, "Amelioration of obesity and glucose intolerance in high-fat-fed C57BL/6 mice by anthocyanins and ursolic acid in Cornelian cherry (Cornus mas)," Journal of Agricultural and Food Chemistry, vol. 54, no. 1, pp. 243-248, 2006.

[18] Y. Lin, M. A. Vermeer, and E. A. Trautwein, "Triterpenic acids present in hawthorn lower plasma cholesterol by inhibiting intestinal ACAT activity in hamsters," Evidence-Based Complementary and Alternative Medicine, vol. 2011, pp. 1-9, Article ID 801272, 2011.

[19] J. Peng, J. Yu, H. Xu et al., "Enhanced liver regeneration after partial hepatectomy in sterol regulatory element-binding protein (SREBP)-1c-null mice is associated with increased hepatocellular cholesterol availability," Cellular Physiology and Biochemistry, vol. 47, no. 2, pp. 784-799, 2018.

[20] D. Haldrup, S. Heebøll, K. L. Thomsen et al., "Preserved liver regeneration capacity after partial hepatectomy in rats with 
non-alcoholic steatohepatitis," World Journal of Hepatology, vol. 10, no. 1, pp. 8-21, 2018.

[21] Y.-R. Jin, J.-l. Jin, C.-H. Li, X.-X. Piao, and N.-G. Jin, "Ursolic acid enhances mouse liver regeneration after partial hepatectomy," Pharmaceutical Biology, vol. 50, no. 4, pp. 523-528, 2012. 